\title{
ANÁLISE DE DESEMPENHO EM REDES DE SENSORES SEM FIO A PARTIR DE UM MEDIDOR DE RSSI
}

\section{PERFORMANCE ANALYSIS IN WIRELESS SENSOR NETWORKS FROM A METER RSSI}

Teles de Sales Bezerra ${ }^{1}$, José Anderson Rodrigues de Souza ${ }^{1}$, Saulo Aislan da Silva Eleuterio ${ }^{1}$, Erlandson de Sales Bezerra ${ }^{2}$ ${ }^{1}$ Discente do Instituto Federal de Educação, Ciência e Tecnologia da Paraíba - IFPB.
${ }^{2}$ Docente do Serviço Nacional de Aprendizagem Industrial da Paraíba - SENAI/PB. E-
mail: teles.tux@gmail.com

RESUMO - Este artigo apresenta a análise de desempenho de um Indicador de Intensidade de Sinal Recebido (RSSI) para Redes de Sensores Sem Fio ZigBee, com a finalidade de investigar o comportamento dos dispositivos ZigBee operando em ambientes reais. $O$ teste de desempenho foi realizado utilizando dois módulos XBee Série 1 e dois módulos de desenvolvimento Arduino Uno R3, avaliando assim a medição de RSSI em um campos de atividades desportivas, no qual o monitoramento de variáveis como umidade e temperatura são importantes.

Palavras-chave: Arduino; Desempenho; ZigBee.

ABSTRACT - This paper presents the performance analysis of a Indicator Received Signal Strength (RSSI) for Wireless Sensor Networks ZigBee, for the purpose of investigating the behavior of ZigBee devices operating in real environments. The performance test was conducted using two modules xbee Series 1 and two Arduino Uno R3 development modules, thus evaluating the RSSI measurement in a sports fields of activity in which the monitoring variables such as humidity and temperature are important.

Keywords: Arduino; Performance; ZigBee.

Recebido em: 05/05/2015 Revisado em: 25/06/2015 Aprovado em: 22/07/2015 


\section{INTRODUÇÃO}

As Redes de Sensores Sem Fio (RSSF) são uma tendência desde os últimos anos devido aos avanços realizados com a comunicação sem fio, as tecnologias de informação e os atributos eletrônicos desenvolvidos para essas tecnologias, Yahaya (2009). As RSSF são uma das mais promissoras dessa geração, devido à usabilidade desta tecnologia em aplicações que vão desde a implementação em sistemas de controles industriais quanto a grande expansão para utilização no cotidiano, o baixo custo de uma RSSF aliada aos sensores multifuncionais que desempenham funções de monitoramento e controle de ações fazem com que a tecnologia seja bastante difundida.

As Redes de Sensores Sem Fio têm atraído um interesse crescente nos últimos anos devido à sua versatilidade de ser empregada em diferentes campos de aplicação, Akyildiz et al. (2002). Em particular nas últimas duas décadas, extensas pesquisas levaram à definição de uma nova geração de sistemas sem fio capazes de ampliar os potenciais campos de aplicação, como nos cuidados da saúde, em casa e na automação em geral. A pergunta mais comum nos sistemas sem fio envolve o alcance dos dispositivos devido à propagação dos sinais de rádio em ambientes internos e externos que são dificultadas pela existência de interferências, Pellegrini (2011).

Uma análise de desempenho e qualidade dos sistemas baseados em RSSF é proposto neste artigo. A tecnologia ZigBee tem crescido vertiginosamente e sua aceitação cresce em ritmo ainda mais acelerado. Os dispositivos ZigBee estão padronizados pelo protocolo IEEE 802.15.4 que tem a finalidade de promover o crescimento e a interoperabilidade entre dispositivos de comunicação, visando a interoperabilidade de tecnologias existentes e aquelas que poderão existir, Ramos (2012).

Diversos trabalhos foram publicados com a finalidade de investigar os efeitos na propagação dos sinais de rádio nos dispositivos ZigBee. Os autores em Hamida e Chelius (2010) investigaram os impactos nos valores de RSSI na movimentação humana em ambientes internos. Os sensores foram utilizados por pesquisadores em andares de um prédio para observar os impactos da atividade humana, mostrando um efeito degradante nos sinais. Em Wadhwa et al. (2009), os impactos na orientação da antena em uma RSSF são mostrados experimentalmente, em que módulos TelosB quando inclinados em direção e sentido contrários causando variações nos valores de RSSI.

Os autores em Graham (2009) monitoraram os efeitos na instalação de 
sensores no teto, onde a antena é invertida e apontada para o chão. Em trabalhos anteriores o foco das pesquisas é sempre voltados aos diferentes fatores que afetam o RSSI, sendo elas a orientação da antena ou o movimento humano, neste trabalho a proposta é que a partir de experimentos possam ser investigados os efeitos causados nos valores de RSSI em ambientes abertos, quando condições como vento, temperatura e umidade são fatores determinantes para a investigação de variações nos sinais de rádio.

O desenvolvimento de um medidor de RSSI é a proposta deste trabalho, em que foram realizadas medições em um campo de práticas desportivas, em que o medidor desenvolvido serviu para realizar diferentes medições em que fatores que influenciam as medições de RSSI foram isolados e com esta análise a propagação dos sinais de rádio foram observadas. O estudo destes dados é de grande importância para entender como é o comportamento de uma RSSF em aplicações como na conservação de espaços para a prática desportiva, em que 0 desperdício de água e o monitoramento da temperatura do solo são importantes fatores na conservação do espaço. Este artigo está organizado da seguinte forma: na Seção 2 explicamos a tecnologia ZigBee e uma descrição do Hardware utilizado na prototipagem do medidor, bem como as características dos módulos XBee, na Seção 3 abordamos os principais conceitos sobre RSSI e a motivação das medições, na Seção 4 apresentamos a metodologia proposta nas medições. Por fim, na Seção 5 e 6 traçamos os resultados nas medições e a apresentação da conclusão do trabalho, respectivamente.

\section{ZIGBEE E DESCRIÇÃO DO HARDWARE}

A tecnologia ZigBee é uma alternativa para atender às aplicações de uso das RSSF. O diferencial dessa tecnologia são as vantagens sobre os demais padrões de comunicação como o Wi-Fi, IEEE (2002), e Bluetooth, IEEE (2003). A tecnologia ZigBee possui um protocolo que suporta redes em malha, estrela e árvore, que dependendo da topologia utilizada um pacote dificilmente deixará de ser entregue.

A tecnologia ZigBee vem ganhando bastante notoriedade por seu código e protocolo simplificado aliado a custos de desenvolvimento reduzidos, além da tecnologia poder integrar-se a partir de módulos para sua construção. O protocolo definido pelo IEEE (Institute of Electrical and Electronics Engineers) para o ZigBee é o IEEE 802.15.4, Saleiro (2009). O ZigBee opera em três segmentos de frequência: $868 \mathrm{MHz}$ na Europa, $915 \mathrm{MHz}$ nos Estados Unidos e 2,4 $\mathrm{GHz}$ em outras localidades. Ele utiliza a definição 802.15.4 do IEEE para implementar as camadas físicas (PHY) e de enlace (MAC). 
As demais camadas do protocolo são definidas pela ZigBee Alliance, ZigBee (2013).

XBee é o nome de uma das tecnologias de módulos utilizados na implementação de hardware necessários em redes ZigBee. A transmissão de pacotes é realizada pela propagação das ondas de rádio por meio da antena, em que transmissão e recepção de dados em sua maioria funcionam a uma potência máxima de 60 $\mathrm{mW}$, e frequências na ordem de 2,4 a 2,48 $\mathrm{GHz}$. Possuem dois modelos: o XBee e $\mathrm{O}$ XBee-Pro.

O Arduino é uma pequena placa de microcontrolador conectado a um computador por meio de uma conexão USB. Além disso, contém diversos outros terminais que permitem a conexão com dispositivos externos, como motores, relés, sensores luminosos, diodos, alto-falantes e outros. 0 projeto da placa é aberto, isso significa que desenvolvedores podem construir placas compatíveis com o Arduino. A plataforma de desenvolvimento Arduino foi o dispositivo base para a construção do protótipo medidor de RSSI, o modelo utilizado no desenvolvimento foi o Arduino Uno R3. A plataforma foi escolhida devido a sua integração facilitada com os módulos XBee.

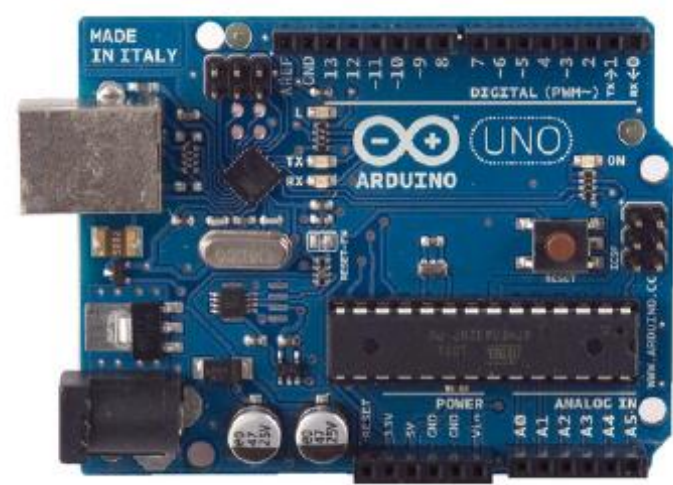

Figura 1. Arduino Uno R3

As placas básicas são complementadas por placas acessórias, denominadas Shields, que podem ser integradas à placa do Arduino. Os Shields são placas de circuito que possuem outros dispositivos (por exemplo, receptores GPS, displays de LCD, módulos de Ethernet etc.) que é conectado ao Arduino para obter funcionalidades adicionais. Dessa forma, um Shield ou módulo de expansão é uma placa de circuito impresso normalmente fixado no topo do Arduino possibilitando a comunicação entre o mesmo e o módulo XBee, através de uma conexão por pinosconectores.

Para o correto acoplamento dos módulos ZigBee ao Arduino foi utilizado um módulo de expansão para Arduino com a padronização para módulos XBee, e o módulo de comunicação XBee S1 MaxStream (Figura 2). Desta forma, o objetivo é o monitoramento do estado do sinal recebido durante as transmissões entre os dois módulos montados da mesma maneira. 


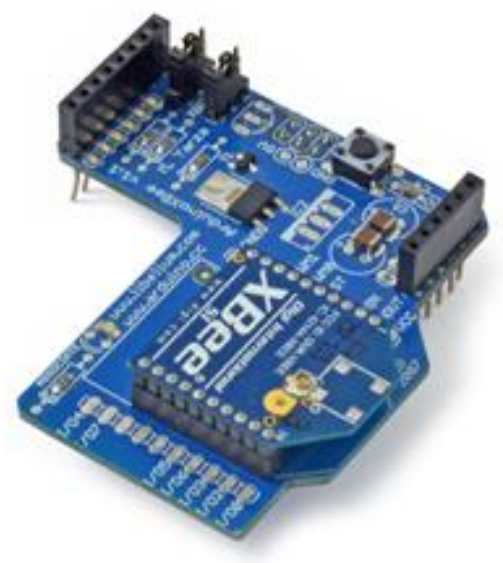

Figura 2. Módulo de Comunicação XBee MaxStream

O Shield XBee permite que uma placa Arduino possa comunicar-se sem fios com outro módulo de tecnologias ZigBee. O módulo utilizado na proposta deste trabalho foi o módulo XBee MaxStream, utilizado como um substituto serial/usb. A configuração dos Shields é realizada por meio de chaves manuais com duas posições, sendo elas Modo XBee e Modo USB, em que posicionada no modo XBee, a chave conecta o pino da saída digital UART (DOUT) do XBee ao terminal RX do microcontrolador, e conecta o pino de entrada de dado UART (DIN) do XBee ao terminal TX do microcontrolador, realizada esta conexão o microcontrolador da plataforma passa a comunicar-se por meio da conexão serial estabelecida na configuração destas chaves.

\section{RSSI}

RSSI (Received Signal Strength Indicator) é uma medida de intensidade de sinal recebido de rádio. Utilizado como uma métrica que estima a qualidade de transmissão entre dois nós a partir da distância entre eles. O RSSI é implementado no padrão IEEE 802.11, Benkic et al. (2008). É um método que utiliza a distância entre o dispositivo emissor e o receptor para designar a qualidade do sinal transmitido utilizando, inclusive, as alterações na intensidade do sinal comparando o sinal recebido com a distribuição de probabilidades e as medidas de localização com base no método de análise estatística, Park (2010). Nas redes de sistemas de comunicação sem fio uma das mais importantes considerações é o efeito de desvanecimento, Lo, Falconer e Sheikh (1994), Chu e Kiang (2004), Wu et al. (2008). Existem diversas formas de desvanecimento da onda eletromagnética afetando consideravelmente a transmissão do sinal entre os nós, como as reflexões em diversos objetos. As ondas de eletromagnetismo viajam por diferentes percursos e distâncias, e a existência e interação de objetos ou barreiras com estas ondas durante o percurso é responsável pelo decremento das distâncias no processo de transmissão e recepção. Os acréscimos ao desvanecimento na propagação da onda eletromagnética são diversos, mas geralmente são atribuídos à reflexão, difração e espelhamento, Wu (2008). 


\section{METOLOGIA DOS EXPERIMENTOS}

Como metodologia para o processo de montagem, desenvolvimento do protótipo e as medições, foram adotadas 4 etapas, sendo elas:

1a Etapa: Nesta etapa foi realizada a montagem do dispositivo medidor de RSSI, em que testes de compatibilidade foram realizados entre a plataforma Arduino Uno R3 utilizada e os módulos XBee, com a finalidade de testar circuitos básicos de acionamento com os módulos e verificar a correta manutenção da comunicação entre os dispositivos. O protótipo foi montado em uma placa de prototipagem, e foram utilizados outros componentes eletrônicos adicionais como um Display LCD 16×2 para a exibição dos valores lidos e componentes para as ligações entre os dispositivos e a alimentação do Display e dos demais dispositivos, como mostrado na Figura 3.

O protótipo dispunha de um terminal de acesso direto à leitura do RSSI, em que um terminal analógico do microcontrolador recebe como entrada o sinal obtido pelo pino PWMO do módulo, que dispõe de um sinal modulado do tipo PWM (Pulse Width Modulation), que é um sinal modulado por largura de pulso e utilizado para transportar um sinal sobre um canal de comunicação, que posteriormente foi realizado 0 tratamento do sinal com a finalidade de obter o valor requerido.

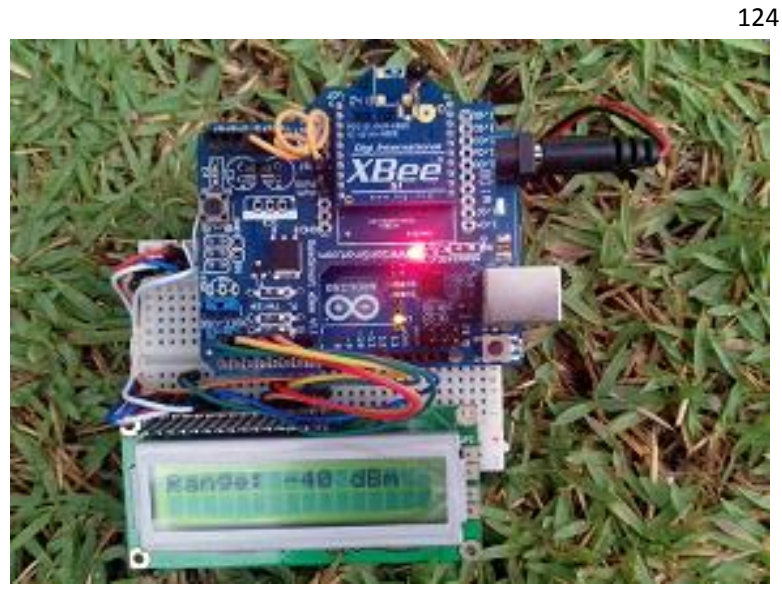

Figura 3. Protótipo de Medidor de RSSI utilizando a plataforma Arduino.

2a etapa: Nesta etapa foi realizado o estudo para a escrita do código-fonte executado pelo protótipo na linguagem Wiring, que é um Framework de programação de código aberto que inclui vários aplicativos que permitem criar com rapidez muitas operações de entrada e saída para microcontroladores, ambos os módulos foram configurados como roteador. Para a medição do valor da entrada analógica que receberá o valor de RSSI foi utilizada a função Pulseln(), própria para este tipo de medição e presente no Framework Wiring. A função lê um pulso (HIGH ou LOW) obtido a partir da leitura do pino PWMO e retornando a duração do pulso em microssegundos. Assim, a função realiza a medição da duração dos pulsos PWM. O código que é executado pelo microcontrolador e realiza a função de leitura é mostrado abaixo, em que a variável dur, por meio da função pulseln(), recebe a duração dos pulsos PWM, e é utilizado na variável rssi com a expressão que retorna o 
valor de RSSI, a implementação da função pulseln() é dada por meio do Framework Wiring.

int rssi;

int dur $=$ pulseIn $(A 1, L O W, 200)$;

rssi $=(\operatorname{dur}+50) *(-1)$;

3a Etapa: As medições foram realizadas em campo aberto utilizado para práticas desportivas, como mostrado na Figura 4, com a fixação do transmissor em um ponto e realizando o deslocamento do receptor ao longo de um trajeto de medições, assim como mostrado na Figura 5.

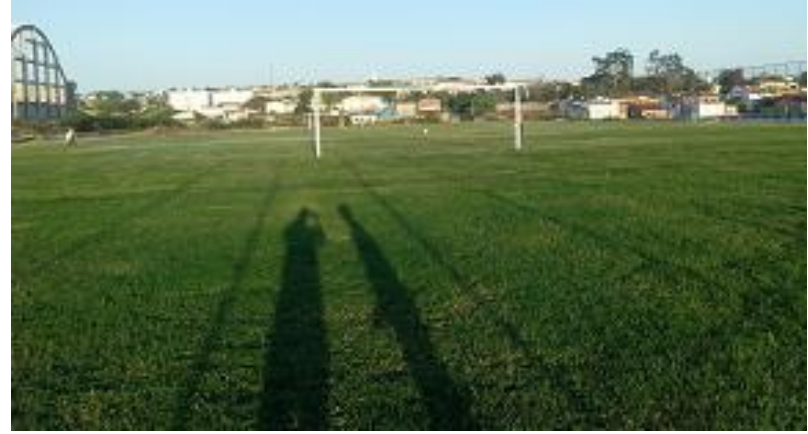

Figura 4. Local onde foram realizadas as medições.

Duas formas de medição foram propostas para a coleta dos dados. No Teste 1 , os dois dispositivos foram colocados em mesmo nível com a grama, com os dois dispositivos ativos e em comunicação. As medições iniciaram no primeiro ponto à uma distância entre ambos de $1 \mathrm{~m}$, então logo em seguida foi distanciado o dispositivo receptor de metro em metro até atingir $7 \mathrm{~m}$, local onde a potência do sinal estava por volta de
$70 \mathrm{dBm}$ de perdas. Para uma maior precisão nas coletas a distância de uma medição para outro foi reduzida para $50 \mathrm{~cm}$, sendo coletados dados até que não existissem mais comunicação entre os módulos. Esse teste foi realizado no horário compreendido entre $14 \mathrm{~h} 30$ e $16 \mathrm{~h} 00$ em um dia com boas condições climáticas, e temperatura ambiente variando entre $31^{\circ} \mathrm{C}$ e $28^{\circ} \mathrm{C}$ e umidade relativa do ar entre $65 \%$ e $60 \%$.

\section{Arranjo das medições}

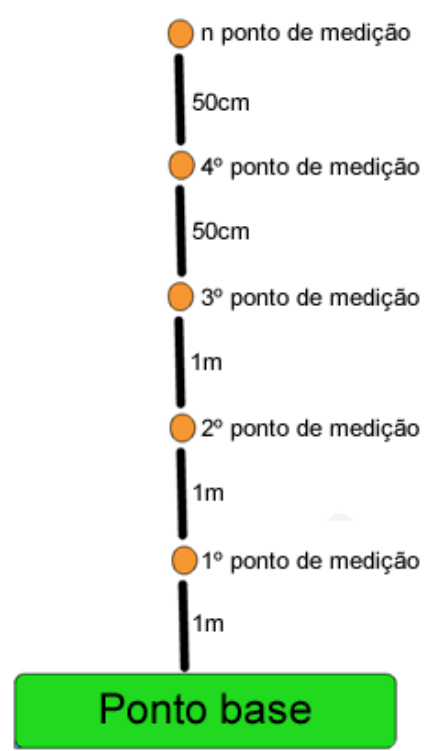

Figura 5. Arranjo da disposição das medições.

No Teste 2, os dispositivos foram dispostos a uma altura de $45 \mathrm{~cm}$, com isso utilizando a mesma metodologia do Teste 1 foram realizadas medições até comprovarmos a não existência de comunicação. Esse teste foi realizado no horário compreendido entre $14 \mathrm{~h} 30$ e $17 \mathrm{~h} 30$ em um dia com boas condições climáticas, e 
temperatura ambiente entre $28^{\circ} \mathrm{C}$ e $24^{\circ} \mathrm{C}$ e umidade relativa do ar entre $55 \%$ e $50 \%$.

4a etapa: Nos testes, para cada ponto 45 amostras de valores de RSSI foram coletadas. Com o fim dos testes e com as amostras coletadas teve início a etapa de processamento das amostras, no primeiro teste obtivemos 13 pontos de medição com 45 amostras coletadas, em que foram calculadas a média, o maior e o menor valor de potência recebida e o desvio padrão. No segundo teste obtivemos 40 pontos de medição com 45 amostras.

\section{RESULTADOS}

A Figura 6 mostra os resultados das medições realizadas, em que à medida que o dispositivo é distanciado a transmissão vai sendo submetida a perdas até não haver mais troca de pacotes, ou seja não havendo mais transmissão, logo entre 9 e $10 \mathrm{~m}$ no Teste 1 não há mais transmissão. Na Figura 7, o gráfico representa os dados obtidos do segundo teste, houve transmissão nesse teste em distâncias maiores que a do teste 1 , a perda de transmissão ocorreu com $26 \mathrm{~m}$.

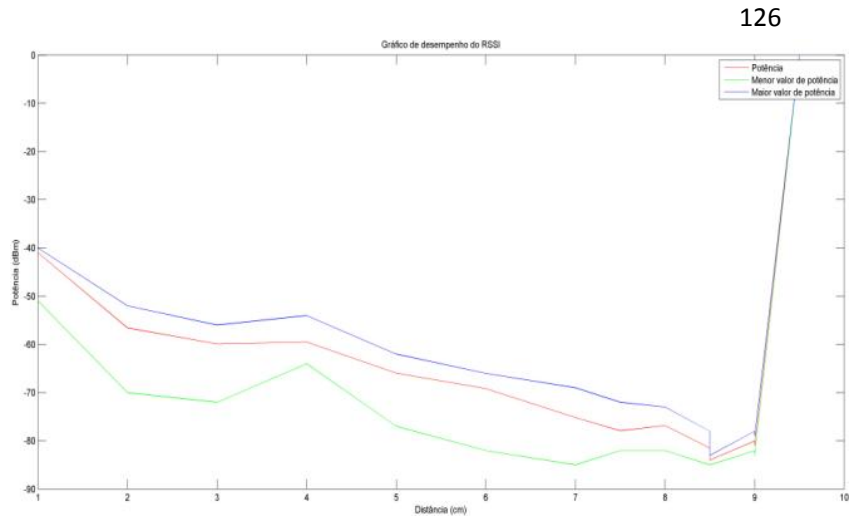

Figura 6. Gráfico da 1a medição realizada no campo.

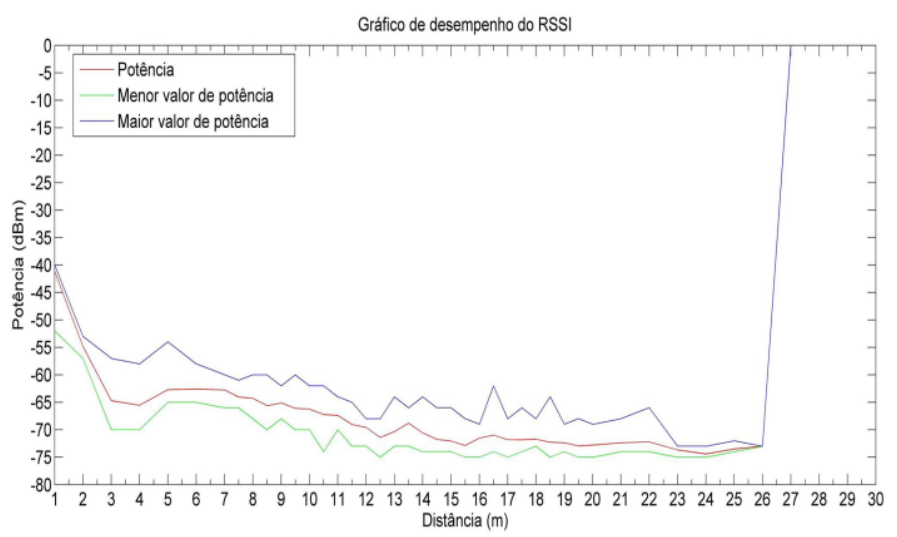

Figura 7. Gráfico da 2a medição realizada no campo.

Analisando os dois gráficos concluímos que no primeiro teste houve transmissão até $9 \mathrm{~m}$ em que a última potência média foi de $-75,95 \mathrm{dBm}$ e no segundo teste houve transmissão até $26 \mathrm{~m}$ onde a última potência média foi de $-73 \mathrm{dBm}$, com isso podemos observar que houve uma grande diferença entre os dois testes. Com esse modelo de dispositivo desenvolvido e com essas condições de temperatura e posicionamento dos dispositivos a transmissão é nula entre $-70 \mathrm{dBm}$ e $-75 \mathrm{dBm}$. 
Em outra análise das amostras foi observado uma dispersão entre as amostras, então para calcular essa dispersão utilizamos desvio padrão (1) para cada distância medida. O desvio padrão é uma medida de dispersão usada com a média. Mede a variabilidade dos valores à volta da média. 0 valor mínimo do desvio padrão é 0 indicando que não há variabilidade, ou seja, que todos os valores são iguais à média. Para seu cálculo, deve-se obter a média da distribuição e, a seguir, determinar os desvios da mesma. Assim, o desvio padrão é a média quadrática dos desvios em relação à média aritmética de uma distribuição, ou seja, é a raiz quadrada da média aritmética dos quadrados dos desvios, esses tomados a partir da média aritmética.

$$
S=\sqrt{\frac{1}{n-1} \sum_{i=n}^{n}\left(x_{i}-\bar{x}\right)^{2}}
$$

Na Figura 8, o gráfico mostra o desvio padrão do teste 1 . Podemos observar que até de $8 \mathrm{~m}$ os valores do desvio ficaram entre $1,31 \mathrm{dBm}$ e $5 \mathrm{dBm}$ isso mostra que até esse ponto não houve grande variação entre os valores de RSSI. Já a partir do ponto de $8,5 \mathrm{~m}$ os valores do desvio padrão foram elevados, como por exemplo 40,26 dBm em 9 m, já que nesses pontos os valores de RSSI tiveram grande variação.

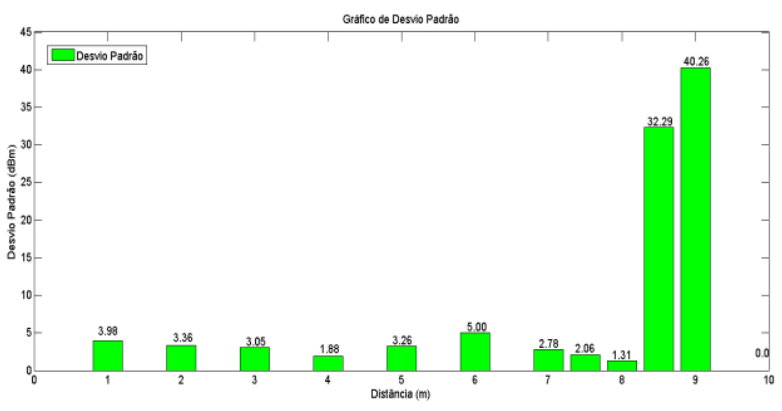

Figura 8. Gráfico do Desvio da 1a medição no campo.

Na Figura 9, o gráfico mostra o desvio padrão do teste 2. Nesse teste podemos observar que também houve grandes diferenças no valor do desvio padrão entre os pontos medidos do ponto 1 até $14 \mathrm{~m}$, os valores do desvio padrão ficaram entre 1,03 $\mathrm{dBm}$ e $3,42 \mathrm{dBm}$. Isso mostra que até esse ponto não houve grande variação entre os valores de RSSI, já do ponto $14,5 \mathrm{~m}$ os valores do desvio padrão foram altos como, por exemplo, 36 e $51 \mathrm{dBm}$ no ponto de 15,5 m já nesses pontos os valores de RSSI variaram muito, assim como observado em $18 \mathrm{~m}$, onde se esperava uma valor alto para o desvio padrão devido a maior influência de interferências como o vento, mas foi obtido o valor de $1,60 \mathrm{dBm}$.

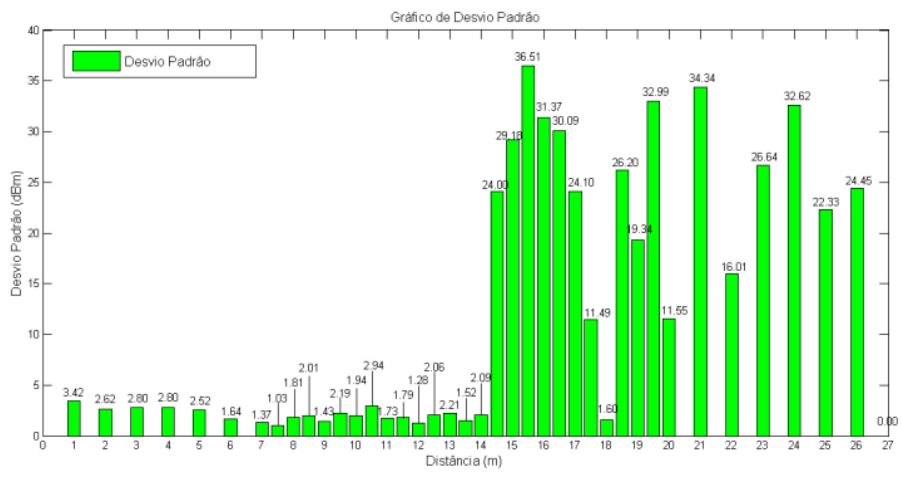

Figura 9. Gráfico do Desvio da 2a medição no campo. 
6 CONSIDERAÇÕES FINAIS

O trabalho desenvolvido e a construção do protótipo tiveram seus objetivos alcançados que foi o de analisar como a intensidade de potência do sinal recebido se comporta em ambientes de campo aberto, em que o valor obtido a partir da leitura do RSSI é um bom indicador para uma boa comunicação, é possível fazer uma consideração também sobre a comprovação de que estes dispositivos devem alcançar a comunicação até 100 metros segundo o fabricante, porém segundo os testes demonstrados aqui essa condição não foi possível ser alcançada assim como descrito nos experimentos. Nossas expectativas foram apoiadas por pesquisas anteriores, e no pressuposto de que, na existência de fatores externos ambientais, os valores de RSSI variem mais do que no cenário apresentado, e de fato observou-se nos dois testes que os valores RSSI variaram bastante, a comprovação dessas variantes obteve-se a partir da extração do desvio padrão em cada ponto de medição.

Nos dois testes a dispersão dos valores foram baixas no início dos testes, porém de um ponto para outro a dispersão aumentou consideravelmente, seguindo na ordem de quanto foi distanciado os nós obtém-se um aumento do desvio, em consequência a transmissão entre os nós se torna instável. Tomando como base esse experimento ganhamos experiência e conhecimentos, onde propomos para trabalhos futuros a realização de medições de sinal RSSI entre prédios, em medições aquáticas e aplicadas à agricultura assim como investigar sobre o uso ou não da criptografia na comunicação dos modems e em que isso iria interferir no alcance.

\section{AGRADECIMENTOS}

Os autores agradecem ao Instituto Federal de Educação Ciência e Tecnologia da Paraíba (IFPB) e ao Conselho Nacional de Desenvolvimento Científico e Tecnológico (CNPq).

\section{REFERÊNCIAS}

AKYILDIZ, I.F. et al. A survey on sensor networks. IEEE, Communications Magazine, v.40, n.8, p.102-114, 2002. http://dx.doi.org/10.1109/MCOM.2002.1024 $\underline{422}$

BENKIC, K. et al. Using RSSI value for distance estimation in wireless sensor networks based on ZigBee. In: SYSTEMS, SIGNALS AND IMAGE PROCESSING. IWSSIP 2008. 15. Proceedings... 2008.

p.303-306. http://dx.doi.org/10.1109/iwssip.2008.46044 27

CHU, J.-L.; KIANG, J.F. Multipath effects on beacon performances. In: NETWORKING, SENSING AND CONTROL. Proceedings... IEEE, 2004. v.1, p.635-638.

GRAHAM, B. et al. Analysis of the effect of human presence on a wireless sensor network. International Journal of Ambient Computing and Intelligence, v.3, n.1, 2011, 
p.1-13.

http://dx.doi.org/10.4018/jaci.2011010101

HAMIDA, B,E.; CHELIUS, G. Investigating the impact of human activity on the performance of wireless networks 2014; An experimental approach. In: WORLD OF WIRELESS MOBILE AND MULTIMEDIA NETWORKS (WOWMOM), IEEE INTERNATIONAL SYMPOSIUM. Proceedings... june 2010. p.1-8.

IEEE Std 802.15.1 IEEE Standard for Information technology-Telecommunications and information exchange between systemsLocal and metropolitan area networksSpecific requirements Part 15.1: Wireless Medium Access Control (MAC) and Physical Layer (PHY) Specifications for Wireless Personal Area Networks (WPANs). IEEE Std. 802.15.1-2002, 2002.

IEEE standard for information technology telecommunications and information exchange between systems - local and metropolitan area networks specific requirements part 15.4: Wireless medium access control (MAC) and physical layer (PHY) specifications for low-rate wireless personal area networks (LR-WPANs). IEEE Std 802.15.4-2003, 2003.

LO, N.W.; FALCONER, D.; SHEIKH, A.U. Adaptive equalization for a multipath fading environment with interference and noise. In: VEHICULAR TECHNOLOGY CONFERENCE. 44. Proceedings... IEEE, 1994. p.252-256. http://dx.doi.org/10.1109/vetec.1994.34512 $\underline{6}$

PARK, C. et al. Localization algorithm design and implementation to utilization RSSI and AOA of ZigBee. In: FUTURE INFORMATION TECHNOLOGY (FUTURETECH). 5. Proceedings... IEEE, 2010. p.1-4. http://dx.doi.org/10.1109/futuretech.2010.5 $\underline{482710}$

PELLEGRINI, R. et al. RF propagation analysis for ZigBee Sensor Network using RSSI measurements. In:

WIRELESS

COMMUNICATION,

VEHICULAR

TECHNOLOGY, INFORMATION THEORY AND

AEROSPACE ELECTRONIC SYSTEMS

TECHNOLOGY (Wireless VITAE).

Proceedings... 2011. p. 1-5. http://dx.doi.org/10.1109/wirelessvitae.2011 .5940834

RAMOS, J.S.B. Instrumentação eletrônica sem fio: transmitindo dados com módulos XBee ZigBee e PIC16F877A. 2012.

SALEIRO, M. ZigBee uma abordagem prática. 2009.

WADHWA, M. et al. The impact of antenna orientation on wireless sensor network performance. In: Computer Science and Information Technology. IEEE International Conference. Proceedings... p.143-147, aug. 2009.

http://dx.doi.org/10.1109/iccsit.2009.523497 8

WU, R.H. et al. Study of characteristics of RSSI signal. In: Industrial Technology, 2008. Proceedings... IEEE, 2008. p.1-3.

YAHAYA, F.H. et al. Performance analysis of wireless sensor network. In: INTERNATIONAL COLLOQUIUM ON SIGNAL PROCESSING \& ITS APPLICATIONS, $5 . \quad$ Proceedings... 2009. http://dx.doi.org/10.1109/cspa.2009.506925 $\underline{9}$

ZIGBEE ALLIANCE. Disponível em: $<$ http://www.zigbee.org>. Acesso em: jun. 2013. 